\title{
A Non-Field Analytical Method for Solving Some Nonlinear Problems in Heat Transfer
}

\section{Vladimir Kulish ( $\nabla$ vladimir.kulish@fs.cvut.cz )}

Czech Technical University in Prague

\section{Pavel Sláma}

Czech Technical University in Prague

\section{Research Article}

Keywords: the method of Kulish, nonlinear heat transfer problems, combustion, ignition, radiative cooling/heating, chemical reactions

Posted Date: May 27th, 2021

DOI: https://doi.org/10.21203/rs.3.rs-561273/v1

License: (1) This work is licensed under a Creative Commons Attribution 4.0 International License. Read Full License 


\title{
A Non-Field Analytical Method for Solving Some Nonlinear Problems in Heat Transfer
}

\author{
Vladimir Kulish ${ }^{*}$, Pavel Sláma1 \\ 1 Department of Thermodynamics \& Fluid Mechanics, Faculty of Mechanical \\ Engineering, Czech Technical University in Prague, Czech Republic (email: \\ vladimir.kulish@fs.cvut.cz) \\ * All correspondence should be addressed to V.K.
}

Keywords: the method of Kulish, nonlinear heat transfer problems, combustion, ignition, radiative cooling/heating, chemical reactions

\begin{abstract}
This paper presents an extension of the non-field analytical method - known as the method of Kulish - to some nonlinear problems in heat transfer. In view of the fact that solving nonlinear problems is very complicated in general, the extension of the method is presented in the form of several important illustrative examples. Two classes of problems are considered: first are the problems, in which the heat equation contains nonlinear terms, while the second type of the problems includes some problems with nonlinear boundary conditions. From the practical viewpoint, the case considered in Section 4 is of the greatest interest. In that section, it is shown that, for complex heat transfer problems, where applications of the non-field method are practically impossible due to a large volume of necessary computations, it is still possible to analyse the solution behaviour and automatically determine similarity criteria for the limiting values of the parameters. Wherever possible the obtained solutions are compared with known solutions obtained by other methods. The practical advantages of the non-field method over other analytical methods are emphasised in each case.
\end{abstract}




\section{Introduction}

Nonlinear heat transfer problems are of much interest in many applications, among which are heat transfer with nonlinear heating or cooling of the boundary (e.g., by radiation), heat transfer due to chemical reactions, ignition and explosions, combustion, as well as many others.

\section{Nomenclature}

\begin{tabular}{|llll|}
\hline$a_{n}$ & coefficients in fractional series & Greek & \\
$C_{1}, C_{2}$ & arbitrary constants & $\alpha, \beta, \gamma$ & arbitrary functions \\
$F$ & intensive property & $\Theta$ & temperature jump \\
$f, g$ & $\begin{array}{l}\text { arbitrary functions of } \\
\text { independent variables }\end{array}$ & $\kappa$ & expansion parameter \\
$Q$ & volumetric heat source & $\lambda, \tau$ & auxiliary variables \\
$q$ & temperature gradient & $v$ & order of fractional differ- \\
& & & integration \\
$T$ & temperature & Special symbols \\
$t$ & time & $\partial^{\nu}$ & fractional differential operator of \\
& & & order $v$ \\
$x$ & spatial variable & $\ell$ & fractional operator \\
$z$ & arbitrary independent variable & $\nabla$ & Laplacian
\end{tabular}

This paper presents an extension of the analytical method for solving energy transport problems, which has been proposed by Kulish and Lage 1 . The method renders the socalled non-field solutions of the energy transport equations (e.g., heat and diffusion equations). These solutions are know as non-field, because they relate local values of the unknown variable (e.g., temperature, mass concentration, velocity) and its spatial derivative. Recently, the generalised procedure and theoretical justification of the method have been reported ${ }^{2}$.

It has been shown that the transport equation in the form

$$
\left[\frac{\partial}{\partial t}-\alpha(x, t) \frac{\partial^{2}}{\partial x^{2}}-\beta(x, t) \frac{\partial}{\partial x}+\gamma(x, t)\right] F=0
$$

can be reduced to an equivalent equation 


$$
\left[\frac{\partial}{\partial t}-\sqrt{\alpha} \ell\left(\frac{1}{\sqrt{\alpha}} \ell\right)+\sqrt{\alpha}\left(\frac{\partial \ell}{\partial x}\right)-\frac{1}{2 \sqrt{\alpha}} \frac{\partial \alpha}{\partial x} \ell+\frac{\beta}{\sqrt{\alpha}} \ell+\gamma\right] F=0
$$

where the operator

$$
\ell=\sum_{n=0}^{\infty} a_{n}(x, t) \partial^{1-n / 2}
$$

is in the form of a series with respect to fractional derivatives $\partial^{1-n / 2}$. In the latter equation, fractional derivatives are defined through the Riemann-Liouville integrals as

$$
\partial^{\nu} f(t)=\frac{d^{\nu} f(t)}{d t^{\nu}}=\frac{1}{\Gamma(-\nu)} \int_{0}^{t} \frac{f(\zeta) d \zeta}{(t-\zeta)^{\nu}}, \operatorname{Re}(\nu)<0
$$

It has been shown then that the expanded form of Eq. (2) is

$$
\begin{array}{r}
{\left[\frac{\partial}{\partial t}-\sqrt{\alpha} \sum_{n=0}^{\infty} \sum_{m=0}^{\infty} \sum_{p=0}^{\infty}\left(\begin{array}{c}
\frac{1-n}{2} \\
p
\end{array}\right) a_{n} \frac{\partial^{p}}{\partial t^{p}}\left(\frac{a_{m}}{\sqrt{\alpha}} \partial^{1-(m+n) / 2-p}\right)+\right]} \\
{\left[+\sqrt{\alpha} \sum_{n=0}^{\infty} \frac{\partial a_{n}}{\partial x} \partial^{(1-n) / 2}-\frac{1}{2 \alpha} \frac{\partial \alpha}{\partial x} \sum_{n=0}^{\infty} a_{n} \partial^{(1-n) / 2}+\frac{\beta}{\sqrt{\alpha}} \sum_{n=0}^{\infty} a_{n} \partial^{(1-n) / 2}+\gamma\right] F=0}
\end{array}
$$

with the recurrent expressions for $a_{n}$ :

$$
\begin{array}{r}
\partial: 1-a_{0}^{2}=0 \\
\partial^{1 / 2}: \sqrt{\alpha} \frac{\partial a_{0}}{\partial x}+\frac{\beta}{\sqrt{\alpha}} a_{0}-\frac{1}{2 \sqrt{\alpha}} \frac{\partial \alpha}{\partial x}-a_{1} a_{0}-a_{0} a_{1}=0 \\
\partial^{0}: \sqrt{\alpha} \frac{\partial a_{1}}{\partial x}+\frac{\beta}{\sqrt{\alpha}} a_{1}-\frac{1}{2 \sqrt{\alpha}} \frac{\partial \alpha}{\partial x} a_{1}-\gamma-a_{2} a_{0}-a_{1}^{2}-a_{0} a_{2}=0 \\
\partial^{(1-k) / 2}: \sqrt{\alpha} \frac{\partial a_{k}}{\partial x}+\frac{\beta}{\sqrt{\alpha}} a_{k}-\frac{1}{2 \sqrt{\alpha}} \frac{\partial \alpha}{\partial x} a_{k}- \\
-\sqrt{\alpha} \sum_{n=0}^{n+2 p} \sum_{p=0}^{\leq k+1}\left(\begin{array}{c}
\frac{1-n}{2} \\
p
\end{array}\right) a_{n} \frac{\partial^{p}}{\partial t^{p}}\left(\frac{a_{k-1-n-2 p}}{\sqrt{\alpha}}\right)=0, k \geq 2
\end{array}
$$


In the following sections, it is shown how the above results can be extended to some nonlinear problems in heat transfer. Two classes of problems are considered: first are the problems, in which the original transport equation, Eq. (1), contains nonlinear terms, while the second type of the problems includes some problems with nonlinear boundary conditions.

From the practical viewpoint, the case considered in Section 4 is of the greatest interest. In that section, it is shown that, for complex heat transfer problems, where applications of the non-field method are practically impossible due to a large volume of necessary computations, it is still possible to analyse the solution behaviour and automatically determine similarity criteria for the limiting values of the parameters (both large and small).

In view of the fact that solving nonlinear problems is very complicated in general, the extension of the method to nonlinear problems is presented here in the form of several important illustrative examples.

\section{Nonlinear heat equation}

Consider the following nonlinear problem

$$
\begin{array}{lll}
\frac{\partial T}{\partial t}-\frac{\partial^{2} T}{\partial x^{2}}+\kappa\left(\frac{\partial T}{\partial x}\right)^{2}=0, & 0<x<\infty, & 0<t<\infty \\
\left.T\right|_{x=0}=T_{s}(t) ; & \left.T\right|_{x=\infty}=0 ; & \left.T\right|_{t=0}=0
\end{array}
$$

It is necessary to determine the temperature gradient at the boundary $q_{s}=(\partial T / \partial x)_{x=0}$.

The latter example is useful, because the exact solution is $k^{2}$ own ${ }^{3}$ in the case of $T_{s}=1$ :

$$
\begin{aligned}
T & =-\frac{1}{\kappa} \ln \left[1-\left(1-e^{-\kappa}\right) \operatorname{erfc}\left(\frac{x}{2 \sqrt{t}}\right)\right] \\
-q_{s} & =\frac{e^{\kappa}-1}{\kappa} \frac{1}{\sqrt{\pi t}}=\left(1+\frac{\kappa}{2}+\frac{\kappa^{2}}{6}+\frac{\kappa^{3}}{24}+\ldots\right) \frac{1}{\sqrt{\pi t}} \\
& \approx\left(1+0.5000 \kappa+0.1667 \kappa^{2}+0.0416 \kappa^{3}+\ldots\right) \frac{1}{\sqrt{\pi t}}
\end{aligned}
$$

The general method of solving the problem given by Eq. (7) is as follows. Equation

(7) becomes identical to Eq. (1) with $\alpha=1, \beta=-\kappa(\partial T / \partial x)$, and $\gamma=0$. Then the 
coefficients $a_{n}$ can be found from Eq. (6). Obviously, in the given case, these coefficients depend on the unknown function $T$, namely:

$$
\begin{aligned}
& a_{1}=\frac{\beta}{2}=-\frac{1}{2} \kappa T^{\prime} \\
& a_{2}=\frac{1}{2}\left(\frac{\partial a_{1}}{\partial x}-a_{1}^{2}\right)=\frac{1}{2}\left(-\frac{1}{2} \kappa T^{\prime \prime}+\frac{1}{4} \kappa^{2}\left(T^{\prime}\right)^{2}\right)
\end{aligned}
$$

where the primes denote derivatives with respect to the spatial variable.

The expression for $a_{2}$ includes $T^{\prime \prime}$. The latter can be found from the original equation as

$$
T^{\prime \prime}=\dot{T}+\kappa\left(T^{\prime}\right)^{2},
$$

where the dot denotes the derivative with respect to time.

Coefficients $a_{n}$ for all $n>2$ can be found from Eq. (6) in the same way as $a_{1}$ and $a_{2}$, while $T^{\prime \prime}$ must be changed as given by Eq. (10). The final expression contains only the first-order derivatives with respect to the spatial variable, but the time derivatives of all orders. That is,

$$
\begin{aligned}
& a_{3}=\frac{1}{8} \kappa^{2} T^{\prime} \dot{T}-\frac{1}{8} \kappa^{3}\left(T^{\prime}\right)^{3} \\
& a_{4}=\frac{1}{16} \kappa \ddot{T}-\frac{3}{32} \kappa^{2} \dot{T}^{2}-\frac{9}{32} \kappa^{3} \dot{T}\left(T^{\prime}\right)^{2}-\frac{25}{128} \kappa^{4}\left(T^{\prime}\right)^{4} \\
& a_{5}=-\frac{1}{32} \kappa^{2} \dot{T} \dot{T}^{\prime}+\frac{1}{32} \kappa^{2} T^{\prime} \ddot{T}-\frac{1}{32} \kappa^{3} \dot{T}^{\prime}-\frac{5}{16} \kappa^{3} T^{\prime} \dot{T}^{2}-\frac{23}{32} \kappa^{4}\left(T^{\prime}\right)^{3} \dot{T}-\frac{13}{32} \kappa^{5}\left(T^{\prime}\right)^{5} \\
& a_{6}=-\frac{1}{32} \kappa \dddot{T}-\frac{1}{32} \kappa^{2}\left(\dot{T}^{\prime}\right)^{2}+\frac{3}{64} \kappa \dot{T} \ddot{T}+O\left(\kappa^{3}, \kappa^{4}, \kappa^{5}, \kappa^{6}\right) \\
& \ldots \ldots
\end{aligned}
$$

Notice that, to arrive at the latter expressions, it was assumed that the mixed derivatives $\partial^{m+n} T /\left(\partial^{m} x \partial^{n} t\right)$ are independent of the order of differentiation with respect to different variables.

Using Eq. (11), it becomes possible to establish an expression for $q_{s}\left(T_{s}\right)$ in the form 


$$
\begin{aligned}
-q_{s} & =\partial^{1 / 2} T_{s}-\frac{1}{2} \kappa q_{s} T_{s}-\left(\frac{1}{4} \kappa \dot{T}_{s}+\frac{1}{8} \kappa^{2} q_{s}^{2}\right) \partial^{-1 / 2} T_{s}+\left(\frac{1}{8} \kappa^{2} q_{s} \dot{T}_{s}-\frac{1}{8} \kappa^{3} q_{s}^{3}\right) \partial^{-1} T_{s}+ \\
& +\left(\frac{1}{16} \kappa \ddot{T}_{s}-\frac{3}{32} \kappa^{2} \dot{T}_{s}^{2}-\frac{9}{32} \kappa^{3} \dot{T}_{s}^{2}-\frac{25}{128} \kappa^{4} q_{s}^{4}\right) \partial^{-3 / 2} T_{s}+ \\
& +\left(-\frac{1}{32} \kappa^{2} \dot{T}_{s} \dot{q}_{s}+\frac{1}{32} \kappa^{2} q_{s} \ddot{T}_{s}-\frac{1}{32} \kappa^{3} \dot{q}_{s}-\frac{5}{16} \kappa^{3} q_{s} \dot{T}_{s}^{2}-\frac{23}{32} \kappa^{4} q_{s}^{3} \dot{T}_{s}-\frac{13}{32} \kappa^{5} q_{s}^{5}\right) \partial^{-2} T_{s}+ \\
& +\left[-\frac{1}{32} \kappa \dddot{T}_{s}-\frac{1}{32} \kappa^{2} \dot{q}_{s}^{2}+\frac{3}{64} \kappa \dot{T}_{s} \ddot{T}_{s}+O\left(\kappa^{3}, \kappa^{4}, \kappa^{5}, \kappa^{6}\right)\right] \partial^{-5 / 2} T_{s}+\ldots
\end{aligned}
$$

In contrast with the corresponding linear problem, the unknown variable is present in both the sides of Eq. (12), which is an ordinary differential equation for $q_{s}$. This differential equation is of an infinite order and, at first sight, looks unsolvable. However, there exists a quite simple way to determine $q_{s}$ upon assuming that the solution is in the form of a power series with respect to the parameter $\kappa$, so that,

$q_{s}=\sum_{n=0}^{\infty} \kappa^{n} q_{n}$

Upon substituting Eq. (13) into (12) and equating the expressions at the same powers of $\kappa$ follow the recurrent expressions for $q_{n}$, namely:

$$
\begin{aligned}
& \kappa^{0}:-q_{0}=\partial^{1 / 2} T_{s} \\
& \kappa^{1}:-q_{1}=-\frac{1}{2} q_{0} T_{s}-\frac{1}{4} \dot{T}_{s} \partial^{-1 / 2} T_{s}+\frac{1}{16} \ddot{T}_{s} \partial^{-3 / 2} T_{s}+\ldots \\
& \kappa^{2}:-q_{2}=-\frac{1}{2} q_{1} T_{s}-\frac{1}{8} q_{0}^{2} \partial^{-1 / 2} T_{s}+\frac{1}{8} q_{0} \dot{T}_{s} \partial^{-1} T_{s}-\frac{3}{32} \dot{T}_{s}^{2} \partial^{-3 / 2} T_{s}+ \\
& +\left(-\frac{1}{32} \dot{T}_{s} \dot{q}_{0}+\frac{1}{32} q_{0} \ddot{T}_{s}\right) \partial^{-2} T_{s}+\left(-\frac{1}{32} q_{0}^{2}+\frac{3}{64} \dot{T}_{s} \ddot{T}_{s}\right) \partial^{-5 / 2} T_{s}+\ldots \\
& \kappa^{3}:-q_{3}=-\frac{1}{2} q_{2} T_{s}-\frac{1}{4} q_{0} q_{1} \partial^{-1 / 2} T_{s}+\left(\frac{1}{8} q_{1} \dot{T}_{s}-\frac{1}{8} q_{0}^{3}\right) \partial^{-1} T_{s}-\frac{9}{32} \dot{T}_{s} q_{0}^{2} \partial^{-3 / 2} T_{s}+ \\
& +\left(-\frac{1}{32} \dot{T}_{s} \dot{q}_{1}+\frac{1}{32} q_{1} \ddot{T}_{s}-\frac{1}{32} \dot{q}_{0} q_{0}^{2}-\frac{5}{16} q_{0} \dot{T}_{s}^{2}\right) \partial^{-2} T_{s}+\ldots
\end{aligned}
$$

Hence, the solution to problem (7) is given by Eqs. (13) and (14).

The solution can be validated by comparing it with the known exact solution for the case when there is a temperature jump at the boundary, that is, $T_{s}=1$. Then Eq. (13) becomes 
$-q_{s}=\left(1+0.50000 \kappa+0.1691 \kappa^{2}+0.0421 \kappa^{3}+\ldots\right) \frac{1}{\sqrt{\pi t}}$

Comparison between Eq. (15) and the exact solution given by Eq. (8) shows that the form of the non-field solution is correct. There is some difference only between the values of the coefficients at various powers of $\kappa$. Thus, the coefficients at $\kappa^{0}$ and $\kappa^{1}$ have been defined exactly, while the coefficients at $\kappa^{2}$ and $\kappa^{3}$ have been defined with the relative errors of $1.4 \%$ and $1.2 \%$, respectively.

In principle, one can look for the solution of the original problem (7) in the form of a power series with respect to $\kappa$ by defining the entire temperature field for each approximation. However, if doing so in practice, it becomes possible to only the first two terms in Eq. (13) - and even then a complicated integral formula must be used. Calculating higher order terms even for a given $T_{s}(t)$ requires a large volume of calculations, which, in practice, cannot be carried on. On the contrary, the non-field method, employed here, allows one to easily calculate several terms of the power series, due to the fact that there is no need to define the entire temperature field for each approximation. The latter circumstance is, beyond any doubt, a major advantage of the non-field method.

To conclude this section, it is worth noting that, with some modifications, the same method becomes applicable for equations with a strong nonlinearity. An extension of the method to this type of problems will be reported in one of the following papers.

\section{Transient heat transfer with a nonlinear heat sink}

The solution obtained in the preceding section has a simple structure and does not provide an idea of some characteristic features of the method. In view of this, it is useful to consider a more complicated example, which possesses all features of an arbitrary nonlinear problem.

Consider a process of heat transfer with a nonlinear heat sink given by $a T+b T^{2}$. Upon choosing proper scales for the variables, the corresponding problem can be written in the form

$$
\begin{array}{lll}
\frac{\partial T}{\partial t}-\frac{\partial^{2} T}{\partial x^{2}}+\kappa\left(T+\frac{T^{2}}{2}\right)=0, & 0<x<\infty, & 0<t<\infty \\
\left.T\right|_{x=0}=T_{s}(t) ; & \left.T\right|_{x=\infty}=0 ; & \left.T\right|_{t=0}=0
\end{array}
$$

The heat sink function has been chosen so that it can represent an exponential dependence with small temperature deviations from the initial value, because $T+T^{2} / 2 \approx \exp (T)-1$. Such a behaviour of the heat sink may be, for example, due to a chemical reaction or radiation with a small temperature difference. 
As in the previous problem, it is necessary to determine the temperature gradient at the boundary $q_{s}=(\partial T / \partial x)_{x=0}$.

Following the same procedure as in Section 2, assume $\alpha=1, \beta=0$, and $\gamma=\kappa(1+T / 2)$ in Eq. (1). Then the relation between the temperature and its gradient can be written in the form analogous with Eq. (12), that is,

$$
\begin{aligned}
-q_{s} & =\partial^{1 / 2} T_{s}+\frac{\kappa}{2}\left(1+\frac{1}{2} T_{s}\right) \partial^{-1 / 2} T_{s}+\frac{\kappa}{8} q_{s} \partial^{-1} T_{s}-\left(\frac{\kappa^{2}}{8}+\frac{\kappa^{2}}{16} T_{s}\right) \partial^{-3 / 2} T_{s}- \\
& -\left(\frac{\kappa}{32} \dot{q}_{s}+\frac{3}{32} \kappa^{2} q_{s}+\frac{\kappa^{2}}{32} T_{s} q_{s}\right) \partial^{-2} T_{s}- \\
& -\left(\frac{\kappa^{2}}{64} \dot{T}_{s}+\frac{\kappa^{2}}{64} \dot{T}_{s} T_{s}+\frac{3 \kappa^{2}}{128} q_{s}^{2}-\frac{\kappa^{3}}{16}-\frac{\kappa^{3}}{64} T_{s}+\frac{3 \kappa^{3}}{128} T_{s}^{2}+\frac{\kappa^{3}}{128} T_{s}^{3}\right) \partial^{-5 / 2} T_{s}+ \\
& +\left(\frac{\kappa}{64} \ddot{q}_{s}+\frac{3 \kappa^{2}}{64} \dot{q}_{s}+\frac{\kappa^{2}}{64} T_{s} \dot{q}_{s}-\frac{\kappa^{2}}{128} \dot{T}_{s} q_{s}+\frac{9 \kappa^{3}}{128} q_{s}-\frac{\kappa^{3}}{64} T_{s}^{2} q_{s}\right) \partial^{-3} T_{s}+ \\
& +(\ldots) \partial^{-7 / 2} T_{s}+\ldots
\end{aligned}
$$

Upon substituting Eq. (13) into (17) and equating the expressions at the same powers of $\kappa$ follow the recurrent expressions for $q_{n}$, namely:

$$
\begin{aligned}
-q_{0} & =\partial^{1 / 2} T_{s} \\
-q_{1} & =\left(\frac{1}{2}+\frac{1}{4} T_{s}\right) \partial^{-1 / 2} T_{s}+\frac{1}{8} q_{0} \partial^{-1} T_{s}-\frac{1}{32} \dot{q}_{0} \partial^{-2} T_{s}+\frac{1}{64} \ddot{q}_{0} \partial^{-3} T_{s}-\ldots \\
-q_{2} & =\frac{1}{8} q_{1} \partial^{-1} T_{s}-\left(\frac{1}{8}+\frac{1}{16} T_{s}\right) \partial^{-3 / 2} T_{s}-\left(\frac{1}{32} \dot{q}_{1}+\frac{3}{32} q_{0}+\frac{1}{32} T_{s} q_{0}\right) \partial^{-2} T_{s}- \\
& -\left(\frac{1}{64} \dot{T}_{s}+\frac{1}{64} \dot{T}_{s} T_{s}+\frac{3}{128} q_{0}^{2}\right) \partial^{-5 / 2} T_{s}+\left(\frac{1}{64} \ddot{q}_{1}+\frac{3}{64} \dot{q}_{0}-\frac{1}{64} T_{s} \dot{q}_{0}-\frac{1}{128} \dot{T}_{s} q_{0}\right) \partial^{-3} T_{s}+ \\
& +\left(\frac{1}{128} \ddot{T}_{s}+\frac{1}{128} \dot{T}_{s}^{2}+\frac{1}{128} T_{s} \ddot{T}_{s}+\frac{7}{256} q_{0} \dot{q}_{0}\right) \partial^{-7 / 2} T_{s}+\ldots \\
-q_{3} & =\frac{1}{8} q_{2} \partial^{-1} T_{s}-\left(\frac{1}{32} \dot{q}_{2}+\frac{3}{32} q_{1}+\frac{1}{32} T_{s} q_{1}\right) \partial^{-2} T_{s}+ \\
& +\left(\frac{1}{16}-\frac{3}{64} q_{0} q_{1}+\frac{1}{64} T_{s}-\frac{3}{128} T_{s}^{2}-\frac{1}{128} T_{s}^{3}\right) \partial^{-5 / 2} T_{s}+ \\
& +\left(\frac{1}{64} \ddot{q}_{2}+\frac{3}{64} \dot{q}_{1}+\frac{1}{64} T_{s} \dot{q}_{1}-\frac{1}{128} \dot{T}_{s} q_{1}+\frac{9}{128} q_{0}-\frac{1}{64} T_{s}^{2} q_{0}\right) \partial^{-3} T_{s}+ \\
& +\left(\frac{7}{256} q_{0} \dot{q}_{1}+\frac{7}{256} q_{1} \dot{q}_{0}+\frac{5}{128} \dot{T}_{s}+\frac{9}{256} T_{s} \dot{T}_{s}+\frac{1}{128} T_{s}^{2} \dot{T}_{s}+\frac{3}{128} q_{0}^{2}-\frac{3}{512} T_{s} q_{0}^{2}\right) \partial^{-7 / 2} T_{s}+\ldots
\end{aligned}
$$


Equations (13) and (18) render the solution of problem given by Eq. (16).

The rest of this section is devoted to finding the temperature gradient at the boundary for the case when the boundary temperature experiences a jump, namely: $T_{s}=\Theta=$ const.

From Eq. (18), it follows that

$$
\begin{aligned}
-q_{0} & =\frac{\Theta}{\sqrt{\pi t}} \\
-q_{1} & =\left[\Theta+\Theta^{2}\left(\frac{1}{2}-\frac{1}{8}-\frac{1}{128}-\frac{1}{512}-\ldots\right)\right] \sqrt{\frac{t}{\pi}} \approx \\
& \approx\left[\Theta+0.365 \Theta^{2}\right] \sqrt{\frac{t}{\pi}}
\end{aligned}
$$

As mentioned in the previous section, in principle, the expression for $q_{1}$ can be obtained by the power series expansion method with respect to $\kappa$. The method yields

$$
-q_{1}=\left[\Theta+\Theta^{2}\left(1-\frac{2}{\pi}\right)\right] \sqrt{\frac{t}{\pi}} \approx\left[\Theta+0.363 \Theta^{2}\right] \sqrt{\frac{t}{\pi}}
$$

Comparison of the latter expression with Eq. (19) shows that the coefficient at $\Theta$ has been defined exactly, while the coefficient at $\Theta^{2}$ differs from the exact value by less than $0.6 \%$.

From Eq. (18) follow expressions for higher order terms in Eq. (13):

$$
\begin{aligned}
& -q_{2}=\left[-0.167 \Theta+(-0.125-0.083+0.055-0.004+\ldots) \Theta^{2}+\right. \\
& \left.+(-0.046+0.018-0.004+0.001-0.001+\ldots) \Theta^{3}\right] \sqrt{\frac{t^{3}}{\pi}} \\
& -q_{3}=\left[0.036 \Theta+(-0.021+0.070+0.008-0.016+\ldots) \Theta^{2}+\right. \\
& \quad+(-0.019+0.036-0.015+0.001-\ldots) \Theta^{3}+ \\
& \left.\quad+(-0.004+0.006-0.002+0.002-\ldots) \Theta^{4}\right] \sqrt{\frac{t^{5}}{\pi}}
\end{aligned}
$$

For example, for $\Theta=1$ : 


$$
-q_{s}=\frac{1}{\sqrt{\pi t}}\left[1+1.365 \kappa t-0.348(\kappa t)^{2}+0.079(\kappa t)^{3}-\ldots\right]
$$

The latter solution can be used for all $\kappa t<1$.

\section{Criteria for modelling}

The algorithm, presented in Sections 2 and 3, allows to find asymptotic solutions without the need to conduct laborious computations. For instance, from Eq. (22) follows that, for a given boundary temperature jump $\Theta$,

$-q_{s} \sim \frac{1}{\sqrt{t}} f(\kappa t)$

where $f$ is an unknown function.

Assume not that is is necessary to find the form of an asymptotic solution for $\Theta \rightarrow \infty$. Because $\Theta$ is a dimensionless quantity, similarity theory, with no further assumptions, yields

$-q_{s} \sim \frac{1}{\sqrt{t}} f(\kappa t, \Theta)$

The method presented here, on the other hand, renders - Eqs. (13) and (21) - the form $-q_{s} \sim \frac{\Theta}{\sqrt{t}} f(\Theta \kappa t)$

which is much more informative than Eq. (24).

It is worth noting here that the asymptotic form for $\Theta \rightarrow 0$ also follows from Eqs. (13) and (21), although, in this case, is readily obtainable from solving the corresponding linear problem.

Finally, consider the general formula for an arbitrary value of $\Theta$ :

$$
-q_{s} \sim \frac{\Theta}{\sqrt{t}}\left[1+\left(a_{1}+b_{1} \Theta\right)(\kappa t)+\left(a_{2}+b_{2} \Theta+c_{2} \Theta^{2}\right)(\kappa t)^{2}+\ldots\right]
$$


Information, which can be recovered from Eq. (26), is not trivial: the function $q_{s} \sqrt{t} / \Theta$ depends on integers powers of $\kappa t$ only and the highest power of $\Theta$ in each term does not exceed the corresponding power of $\kappa t$.

Assume now that, for the original problem given by Eq. (16), with $\kappa<0$, it is necessary to determine the ignition time, $t_{\text {ign }}$, which implies $q_{s}\left(t_{\text {ign }}\right)=0$. It follows from Eq. (26) that

$t_{\text {ign }} \approx \frac{C_{1}}{\kappa}, \quad \Theta \rightarrow 0$

$t_{\text {ign }} \sim \frac{\mathrm{C}_{2}}{\kappa \Theta}, \quad \Theta \rightarrow \infty$

Hence, for an arbitrary value of $\Theta$, it becomes possible to offer an interpolation formula

$t_{\text {ign }}=\frac{C_{1}}{\kappa}\left(1+\frac{\Theta}{C_{2}}\right)^{-1}$.

The constant coefficient $C_{1}$ can be found from the corresponding linear problem, for which the term $T^{2} / 2$ is absent. The constant coefficient $C_{2}$ is to be found either by the presented method, or numerically, or from experimental data.

As the concluding example in this section consider the case when the thermal conductivity varies with temperature linearly, namely:

$$
\begin{array}{lll}
\frac{\partial T}{\partial t}-(a+b T) \frac{\partial^{2} T}{\partial x^{2}}-b\left(\frac{\partial T}{\partial x}\right)^{2}=0, & 0<x<\infty, & 0<t<\infty \\
\left.T\right|_{x=0}=T_{s}(t) ; & \left.T\right|_{x=\infty}=0 ; & \left.T\right|_{t=0}=0
\end{array}
$$

The latter problem is equivalent to Eq. (1), in which $\alpha=a+b T, \beta=b(\partial T / \partial x)$, and $\gamma=0$. The relationship between the boundary values of $T_{s}$ and $q_{s}$ is then

$$
\begin{gathered}
-q_{s} \sqrt{a+b T_{s}}=\left\{\partial^{1 / 2}+\frac{1}{4} \frac{b}{\sqrt{a+b T_{s}}} q_{s}+\left(\frac{1}{4} \frac{b}{a+b T_{s}} T_{s}-\frac{5}{32} \frac{b^{2}}{a+b T_{s}} q_{s}^{2}\right) \partial^{-1 / 2}+\right. \\
\left.+\left[\frac{1}{16} \frac{b}{\sqrt{a+b T_{s}}} \dot{q}_{s}-\frac{7}{32} \frac{b^{2}}{\left(a+b T_{s}\right)^{3 / 2}} q_{s} \dot{T}_{s}+\frac{15}{64} \frac{b^{3}}{\left(a+b T_{s}\right)^{3 / 2}} q_{s}^{3}\right] \partial^{-1}+\ldots\right\} T_{s}
\end{gathered}
$$

For the boundary condition of a temperature jump, $T_{s}=\Theta=$ const, the latter equation becomes 


$$
\begin{aligned}
-q_{s} \sqrt{a+b \Theta} & =\frac{\Theta}{\sqrt{\pi t}}+\frac{\Theta}{4} \frac{b}{\sqrt{a+b \Theta}} q_{s}-\frac{5 \Theta}{16 \sqrt{\pi}} \frac{b^{2}}{a+b \Theta} q_{s}^{2} \sqrt{t}+ \\
& +\left[\frac{\Theta}{16} \frac{b}{\sqrt{a+b \Theta}} \dot{q}_{s}+\frac{15 \Theta}{64} \frac{b^{3}}{(a+b \Theta)^{3 / 2}} q_{s}^{3}\right] t+\ldots
\end{aligned}
$$

If only the first term in the right side of the latter equation is taken into account, an approximate expression for $q_{s}$ becomes

$$
-q_{s} \approx \frac{\Theta}{\sqrt{\pi t}} \frac{1}{\sqrt{a+b \Theta}} .
$$

A second approximation follows from substituting Eq. (32) into the second term in the right side of Eq. (31) and truncating all the subsequent terms

$$
-q_{s} \approx \frac{\Theta}{\sqrt{\pi t}} \frac{1}{\sqrt{a+b \Theta}}\left(1-\frac{1}{4} \frac{b \Theta}{a+b \Theta}\right) .
$$

Continuing this iteration process without calculating exact values of the coefficients, one arrives at the expression for $q_{s}$ in the form of power series with respect to $z=b \Theta /(a+b \Theta)$, that is,

$$
-q_{s}=\frac{\Theta}{\sqrt{\pi t}} \frac{1}{\sqrt{a+b \Theta}}\left(1-\kappa_{1} z-\kappa_{2} z^{2}-\ldots\right) .
$$

Approximate values of the coefficients $\kappa_{n}$ can be obtained by substituting the latter expression into Eq. (31). This is beyond the scope of this example, the main aim of which is to show that $q_{s}$ depends on $\Theta$ and $t$ in the form

$$
-q_{s}=\frac{\Theta}{\sqrt{\pi t}} \frac{1}{\sqrt{a+b \Theta}} f\left(\frac{b \Theta}{a+b \Theta}\right) \text {, }
$$

where $f$ is an unknown function of $z$.

If similarity theory is used to tackle the same problem, the following result can be found 
$-q_{s}=\frac{\Theta}{\sqrt{\pi a t}} g\left(\frac{b \Theta}{a}\right)$,

because $q_{s} \sqrt{a t}$ and $b \Theta / a$ are the two dimensionless combinations of the parameters involved. In the latter expression, $g$ is an unknown function of $b \Theta / a$.

However, Eq. (35) is more informative than Eq. (36) due to the following reasons. From considering the limiting cases, it follows that $f(0)=1, \lim _{z \rightarrow 1} f(z)=f(1)=$ const and $0 \leq f(1)<1$. The last inequality can be established from comparing with the solution to the auxiliary problem, in which the thermal conductivity remains constant and equals $b \Theta$.

In the same way, for the function $g$, it follows $g(0)=1$ and $g(\infty)=0$.

Hence, both the functions change within finite limits: $f$ changes within the finite interval $0 \leq z<1$, while $g$ changes within the semi-infinite interval $0 \leq b \Theta / a<\infty$. Due to this difference, the procedure of finding $f$ from additional calculations or experimental data is much simpler than the procedure of finding $g$.

\section{Heat transfer with nonlinear effects on the surface}

In this section, two examples of heat transfer with nonlinear effects on the surface are considered, namely: surface radiative cooling and heating, respectively.

In the first case, the boundary value problem is in the form

$$
\begin{aligned}
& \left(\frac{\partial}{\partial \tau}-\frac{\partial^{2}}{\partial x^{2}}\right) T=0, \quad 0<x<\infty, \quad 0<\tau<\infty \\
& q_{s}=\left.\frac{\partial T}{\partial x}\right|_{x=0}=\lambda T_{s}^{4} ;\left.\quad T\right|_{x=\infty}=\left.T\right|_{\tau=0}=T_{0}=\mathrm{const}
\end{aligned}
$$

where $\tau=\kappa t /\left(\rho c_{p}\right)$ and $\lambda=\sigma / \kappa$

For the given problem, it is necessary to determine the temperature evolution, $T_{s}(\tau)$, on the surface $x=0$.

Notice that a similar problem with $q_{s}=\lambda T_{s}^{2}$ was considered earlier as an illustrative example ${ }^{2}$. In the given case, the equation for the surface temperature is in the form (compare this with Eq. (26) in [2])

$T_{s}(\tau)=T_{0}-\lambda \partial^{-1 / 2} T_{s}^{4}(\tau)$ 
As in the previous study ${ }^{2}$, the solution is sought in the form

$T=\sum_{n=0}^{\infty} a_{n} \tau^{n / 2}, \quad a_{n}=$ const.

Then, by complete analogy with the case $q_{s}=\lambda T_{s}^{2}$, the solution becomes

$$
\frac{T_{s}}{T_{0}}=1-\frac{2}{\sqrt{\pi}}\left(\lambda T_{0}^{3}\right) \tau^{1 / 2}+4\left(\lambda T_{0}^{3}\right)^{2} \tau-\left(\frac{4}{3 \sqrt{\pi}}+\frac{2}{\pi^{3 / 2}}\right)\left(\lambda T_{0}^{3}\right)^{3} \tau^{3 / 2}+\ldots
$$

For practical calculations, the latter expression is valid for small values of $\tau$.

The asymptotic solution for $\tau \rightarrow \infty$ can be easily obtained in the same way as for the case $q_{s}=\lambda T_{s}^{2}$, that is,

$T_{s} \approx\left[T_{0} /(\lambda \sqrt{\pi})\right]^{1 / 4} \tau^{-1 / 8}$

Now consider the case of radiative surface heating by an external volumetric heat source of the constant intensity $Q$. The heat transfer process at the surface is governed by the Stefan-Boltzmann law. It is necessary to determine the temperature evolution, $T_{s}(\tau)$, on the surface $x=0$.

In the given case, the boundary value problem becomes

$$
\begin{aligned}
& \left(\frac{\partial}{\partial \tau}-\frac{\partial^{2}}{\partial x^{2}}\right) T=Q, \quad 0<x<\infty, \quad 0<\tau<\infty \quad Q=\mathrm{const} \\
& \left.\frac{\partial T}{\partial x}\right|_{x=0}=\lambda T_{s}^{4} ;\left.\quad T\right|_{x=\infty}=Q \tau,\left.\quad T\right|_{\tau=0} 0
\end{aligned}
$$

Upon eliminating $(\partial T / \partial x)_{x=0}$ from the boundary condition, the equation for $T_{s}$ follows:

$$
\lambda T_{s}^{4}+\partial^{1 / 2} T_{s}=2 Q \sqrt{\frac{\tau}{\pi}}
$$

Upon substituting Eq. (43) into Eq. (39) and equating the coefficients at equal powers of $\tau$, the solution becomes 
$T_{s}=Q \tau-\lambda Q^{4} \frac{\Gamma(5)}{\Gamma(11 / 2)} \tau^{9 / 2}+\lambda^{2} Q^{7} \frac{4 \Gamma(17 / 2) \Gamma(5)}{\Gamma(9) \Gamma(11 / 2)} \tau^{8}-\ldots$

For practical calculations, the latter expression is valid for small values of $\tau$.

From the physics of the problem, it is clear that, at $\tau \rightarrow \infty$, the value of $T_{s}$ increases slower than $\tau$. In view of this, the term $\partial^{1 / 2} T_{s}$ can be neglected in comparison with $2 Q \sqrt{\tau / \pi}$. Then, the asymptotic solution for large values of $\tau$ becomes

$T_{s} \approx[2 Q /(\lambda \sqrt{\pi})]^{1 / 4} \tau^{1 / 8}$

\section{General discussion}

Some attempts to extend the method of Kulish to nonlinear problems have been made earlier ${ }^{4}$. In particular, the Burgers and Frank-Kamenetskii equations were considered. However, the reported solutions are not completely in a non-field form - a narrow interval within the field is needed to express the corresponding gradient.

The approach, presented in this work, on the other hand, allows not only to extend the method of Kulish to a wide class of nonlinear heat transfer problems, but also obtain non-field solutions, which relate local values of the temperature and its gradient. In most of the cases, these solutions cannot be obtained by other analytical methods, or the procedures leading to these solutions are too laborious (e.g., involve a large volume of calculations), which makes them impractical.

Last but not least, it has been shown that, for complex heat transfer problems, where applications of the non-field method are practically impossible due to a large volume of necessary computations, it is still possible to analyse the solution behaviour and automatically determine similarity criteria for the limiting values of the parameters.

In the following study, the method presented here will be extended, with some modifications, to problems with strong nonlinearities.

\section{References}

1. Kulish, V. V. \& Lage, L. J. Fractional-diffusion solutions for transient local temperature and heat flux. J. Heat Transfer. 122(2), 372-376. https://doi.org/ 10.1115/1.521474 (2000).

2. Kulish, V. A non-field analytical method for solving energy transport equations. $J$. Heat Transfer. 142(4), 042102. https://doi.org/10.1115/1.4046301 (2020).

3. Hahn, D.W. \& Özisik, M.N. Heat Conduction (Wiley, New York, 2012).

4. Novozhilov, V. \& Kulish, V. Extension of relations between local values of intensive properties and corresponding fluxes to some nonlinear processes. $J$. MESA. 5(1), 97-109 (2014). 


\section{Acknowledgments}

The authors would like to thank the Department of Thermodynamics \& Fluid Mechanics of the Faculty of Mechanical Engineering at the Czech Technical University in Prague for the financial support.

\section{Author Contributions}

Both the authors equally contributed into this study.

\section{Additional Information}

Competing financial interests: The authors declare no competing financial interests. 